\title{
Correction to: A review of predictive policing from the perspective of fairness
}

\section{Kiana Alikhademi ${ }^{1}$ (D) Emma Drobina ${ }^{1} \cdot$ Diandra Prioleau $^{1}$. Brianna Richardson ${ }^{1}$. Duncan Purves ${ }^{2}$. Juan E. Gilbert ${ }^{1}$}

Published online: 28 August 2021

(c) Springer Nature B.V. 2021

\section{Correction to: Artifcial Intelligence and Law https://doi.org/10.1007/s10506-021-09286-4}

In the original publication of this article the funding information was missed to publish and the same has been provided here.

Funding This material is based upon work supported by the National Science Foundation under Grant No. 1917712.

The original articles can be found online at https://doi.org/10.1007/s10506-021-09286-4.

\author{
Kiana Alikhademi \\ kalikhademi@ufl.edu \\ Emma Drobina \\ edrobina@ufl.edu \\ Diandra Prioleau \\ dprioleau@ufl.edu \\ Brianna Richardson \\ richardsonb@ufl.edu \\ Duncan Purves \\ dpurves@ufl.edu \\ Juan E. Gilbert \\ juan@ufl.edu
}

1 Computer \& Information Sciences \& Engineering Department, College of Engineering, University of Florida, Gainesville, FL, USA

2 Department of Philosophy, College of Liberal Arts and Sciences, University of Florida, Gainesville, FL, USA 
Publisher's Note Springer Nature remains neutral with regard to jurisdictional claims in published maps and institutional affiliations. 